\title{
DEMICONTINUITY AND HEMICONTINUITY IN FRÉCHET SPACE
}

\author{
HUGO D. JUNGHENN
}

ABSTRACT. It is proved that the notions of demicontinuity and hemicontinuity for monotone maps from a Fréchet space into its dual are equivalent, thus generalizing a result of $\mathrm{T}$. Kato.

Let $X$ be a (real or complex) locally convex Hausdorff linear topological space, $X^{*}$ its dual, and $($,$) the natural pairing between X$ and $X^{*}$. In what follows we consider (possibly) nonlinear operators $G$ with domain $D(G)$ contained in $X$ and range contained in $X^{*}$.

DEFINITIONS. $G$ is said to be

(a) monotonic if $\operatorname{Re}(x-y, G x-G y) \geqq 0 ; x, y \in D(G)$;

(b) demicontinuous at $u \in D(G)$ if $u_{n} \in D(G), n=1,2, \cdots$, and $u_{n} \rightarrow u$ imply $G u_{n} \rightarrow G u(\rightarrow$ and $\rightarrow$ denote strong convergence in $X$ and weak* convergence in $X^{*}$ respectively);

(c) hemicontinuous at $u \in D(G)$ if $v \in X, t_{n}>0, n=1,2, \cdots, t_{n} \rightarrow 0$ and $u+t_{n} v \in D(G)$ imply $G\left(u+t_{n} v\right) \rightarrow G(u)$.

The following theorem generalizes a result of T. Kato (see [1]).

THEOREM. If $X$ is a Fréchet space, $G$ is monotonic, and $D(G)$ is open in $X$, then $G$ is demicontinuous at $u \in D(G)$ if and only if $G$ is hemicontinuous at $u$.

Proof. The necessity is clear. Assume $G$ is hemicontinuous at $u \in$ $D(G)$, and let $u_{n} \in D(G), n=1,2, \cdots, u_{n} \rightarrow u$. We shall show first that $\left\{G u_{n}\right\}$ is a strongly bounded subset of $X^{*}$.

Suppose that this is not the case. Then by the principle of uniform boundedness (see [2]) there exists some $x \in X$ and a subsequence of $\left\{u_{n}\right\}$, which we shall denote by $\left\{u_{n}\right\}$, such that

$$
r_{n}=\left|\left(x, G u_{n}\right)\right| \rightarrow \infty .
$$

We construct a sequence of integers $k_{n}$ as follows:

$$
\begin{aligned}
& k_{n}=\left[\min \left\{\left\|u_{n}-u\right\|^{-1 / 4}, r_{n}\right\}\right] \text { if } u_{n} \neq u \text {, } \\
& =\left[r_{n}\right] \quad \text { if } u_{n}=u,
\end{aligned}
$$

Received by the editors June 22, 1972.

AMS (MOS) subject classifications (1970). Primary 47H05; Secondary 46A05.

Key words and phrases. Fréchet space, demicontinuity, hemicontinuity, monotonic.

(C) American Mathematical Society 1973 
where [ $\cdot$ ] denotes the greatest integer function and $\|\cdot\|$ denotes the quasinorm of $X$. Clearly $k_{n} \rightarrow \infty$ and $\left\|k_{n}^{2}\left(u_{n}-u\right)\right\| \leqq k_{n}^{2}\left\|u_{n}-u\right\| \leqq\left\|u_{n}-u\right\|^{1 / 2}$ for all $n$. Setting $t_{n}=k_{n}^{-1}$ we thus have

$$
t_{n} \rightarrow 0 \text { and } t_{n}^{-2}\left(u_{n}-u\right) \rightarrow 0 .
$$

Let $v \in X$ and set $w_{n}=u+t_{n} v$. Since $D(G)$ is open, $w_{n} \in D(G)$ for all $n$ greater than some $n_{0}$. The monotonicity of $G$ implies that

$$
\operatorname{Re}\left(v, G u_{n}\right) \leqq t_{n}^{-1} \operatorname{Re}\left(w_{n}-u_{n}, G w_{n}\right)+t_{n}^{-1} \operatorname{Re}\left(u_{n}-u, G u_{n}\right) .
$$

By the hemicontinuity of $G,\left\{G w_{n}: n>n_{0}\right\}$ is pointwise bounded and therefore, by the uniform boundedness theorem, equicontinuous. Since $t_{n}^{-1}\left(w_{n}-u_{n}\right) \rightarrow v$ it follows that $\left\{t_{n}^{-1} \operatorname{Re}\left(w_{n}-u_{n}, G w_{n}\right): n>n_{0}\right\}$ is bounded.

Next, we obtain an upper bound for the second term on the right side of (2). Let $p$ denote the continuous seminorm on $X^{*}$ defined by the bounded subset of $X$ consisting of the point $x$ and the sequence $\left\{t_{n}^{-2}\left(u_{n}-u\right)\right\}$. Setting $s_{n}=p\left(G u_{n}\right)$ we have, for all $n$,

Therefore,

$$
t_{n}^{-1} \operatorname{Re}\left(u_{n}-u, G u_{n}\right) \leqq s_{n} t_{n}
$$

$$
\operatorname{Re}\left(v, G u_{n}\right) \leqq C+s_{n} t_{n}, \quad n>n_{0},
$$

where $C$ is a constant depending on $v$ but not on $n$. Dividing by $s_{n} t_{n}$ and noting that $s_{n} t_{n} \geqq r_{n} t_{n} \geqq 1$ we obtain

$$
\operatorname{Re}\left(v,\left(s_{n} t_{n}\right)^{-1} G u_{n}\right) \leqq C+1, \quad n>n_{0} .
$$

Replacing $v$ by $-v$ (and by $\pm i v$ if $X$ is complex) we see that $\left\{\left(v,\left(s_{n} t_{n}\right)^{-1} G u_{n}\right)\right\}$ is bounded for all $v \in X$. By the uniform boundedness theorem again, $\left\{\left(s_{n} t_{n}\right)^{-1} G u_{n}\right\}$ is bounded in $X^{*}$. But this is clearly impossible since $p\left(\left(s_{n} t_{n}\right)^{-1} G u_{n}\right)=t_{n}^{-1} \rightarrow \infty$. Thus $\left\{G u_{n}\right\}$ is bounded.

We now show that $G u_{n} \rightarrow G u$. Define a sequence of integers $j_{n}$ by

$$
\begin{aligned}
j_{n} & =\left[\left\|u_{n}-u\right\|^{-1 / 4}\right] & & \text { if } u_{n} \neq u, \\
& =n & & \text { if } u_{n}=u .
\end{aligned}
$$

If $v \in X$ and we set $t_{n}=j_{n}^{-1}, w_{n}=u+t_{n} v$, then (1) and (2) hold as before. Let $q$ be the continuous seminorm on $X^{*}$ defined by the bounded set $\left\{t_{n}^{-2}\left(u_{n}-u\right)\right\}$ and let $q_{n}=q\left(G u_{n}\right)$. Then $\left\{q_{n}\right\}$ is bounded and

$$
t_{n}^{-1} \operatorname{Re}\left(u_{n}-u, G u_{n}\right) \leqq q_{n} t_{n} \rightarrow 0 .
$$

The hemicontinuity of $G$ implies that $\left\{G w_{n}\right\}$ is equicontinuous, hence

$$
t_{n}^{-1} \operatorname{Re}\left(w_{n}-u_{n}, G w_{n}\right) \rightarrow \operatorname{Re}(v, G u) .
$$


Thus from (2), (3), and (4) we obtain

$$
\lim \sup \operatorname{Re}\left(v, G u_{n}-G u\right) \leqq 0 .
$$

Since $v$ was arbitrary,

$$
\lim \sup \left|\left(v, G u_{n}-G u\right)\right|=0 \text { for all } v \in X,
$$

hence $G u_{n} \rightarrow G u$.

\section{REFERENCES}

1. T. Kato, Demicontinuity, hemicontinuity, and monotonicity. II, Bull. Amer. Math. Soc. 73 (1967), 886-889. MR 38 \#6411.

2. K. Yosida, Functional analysis, Die Grundlehren der math. Wissenschaften, Band 123, Academic Press, New York; Springer-Verlag, Berlin, 1965. MR 37 \#725.

Department of Mathematics, George Washington University, Washington, D.C. 20006 